\title{
C allele of angiotensin II type 1 receptor gene A1166C polymorphism affects plasma adiponectin concentrations in healthy young Japanese women
}

\begin{abstract}
Katsuko Miyanaga $^{1,2}$, Keisuke Fukuo ${ }^{1,2}$, Hiroshi Akasaka ${ }^{3}$, Tomohiro Katsuya ${ }^{4}$, Rumi Fukada ${ }^{1}$, Hiromi Rakugi $^{4}$ and Tsutomu Kazumi ${ }^{1}$

Angiotensin II and its type 1 receptor (AT1R) are both expressed in the adipose tissue and involved in the genesis of atherosclerosis as well as hypertension. However, the influence of the AT1R gene A1166C polymorphism on atherosclerosis risk factors and on the development of early atherosclerosis is not clear. We evaluated 416 healthy young women to investigate the effects of this genotype on atherosclerosis risk factors and on carotid intima-media thickness as a validated marker of early atherosclerosis. After adjusting for confounding factors including body mass index, homeostasis model assessment of insulin resistance and plasma high-density lipoprotein (HDL)-cholesterol levels, plasma adiponectin concentrations were significantly lower in carriers of the $\mathrm{C}$ allele compared with non-carriers. Moreover, multiple logistic regression analysis showed that the $C$ allele was the strongest and most independent determinant of lower plasma adiponectin concentrations. It is noted that the participants with the lowest quartile of plasma adiponectin concentrations had thicker levels of carotid intima-media thickness, lower plasma HDL-cholesterol and lipoprotein lipase levels, as well as higher trunk fat mass compared with the highest quartile. In addition, a weak but significant positive correlation was observed between percentages of fat in the diet and plasma adiponectin concentrations in non-carriers of the C allele. In conclusion, AT1R A1166C was associated with plasma adiponectin concentrations and influenced the correlations between dietary fat intake and plasma concentrations of adiponectin. These findings may help to identify vulnerable populations that are susceptible to the development of atherosclerosis and require early dietary recommendations for young women.
\end{abstract}

Hypertension Research (2009) 32, 901-905; doi:10.1038/hr.2009.111; published online 21 August 2009

Keywords: angiotensin II type 1 receptor; polymorphism; adiponectin; dietary fat intake

\section{INTRODUCTION}

Accumulating evidence shows that angiotensin II (AII), a major effector peptide of the renin-angiotensin system (RAS), contributes to the pathogenesis of the metabolic syndrome and atherosclerosis. ${ }^{1-3}$ It is noted that human adipose tissue possesses all of the components necessary for production of AII and AII type 1 receptor (AT1R). The adipose tissue RAS has a role in the process of adipogenic differentiation and in the regulation of body weight. ${ }^{3}$ Most physiological responses triggered by AII occur through activation of the G-protein-coupled AT1R. A common polymorphism in the AT1R (A1166C) has been linked to enhanced physiological responses to $\mathrm{AII}{ }^{4}$ In addition, this polymorphism in the human $A T 1 R$ has been associated with phenotypic effects including insulin sensitivity and metabolic syndrome traits as well as high blood pressure. ${ }^{5,6}$ Although this polymorphism is located in the $3^{\prime}$-untranslated region of the human $A T 1 R$, recent evidence shows that the polymorphism can lead to increased AT1R densities through inhibition of the ability of miR-155 to attenuate translation of AT1R mRNA. ${ }^{7}$

Adipose tissue is a highly active metabolic and endocrine organ that secretes many biologically active substances, collectively known as adipocytokines. ${ }^{8}$ Adiponectin is an anti-atherogenic and insulinsensitizing adipocytokine that is stably present in the plasma at very high concentrations. Human studies showed that adiponectin is downregulated in patients with obesity-related diseases, including type 2 diabetes, metabolic syndrome and hypertension. ${ }^{9}$ Importantly, AII infusion decreases plasma adiponectin levels, ${ }^{10}$ and RAS blockade decreases adipocyte size with improvement in insulin sensitivity in rats. ${ }^{11}$ In addition, blockade of the RAS increases plasma adiponectin concentrations in patients with essential hypertension. ${ }^{12}$ However, it remains to be determined whether AT1R A1166C affects plasma adiponectin concentrations in humans. Furthermore, plasma adiponectin concentrations were lower in mice fed a high-fat diet compared

${ }^{1}$ Department of Food Sciences and Nutrition, School of Human Environmental Sciences, Mukogawa Women's University, Nishinomiya, Japan; ${ }^{2}$ The Research Center for Elderly Nutrition and Development, Mukogawa Women's University, Nishinomiya, Japan; ${ }^{3}$ Second Department of Internal Medicine, Sapporo Medical University School of Medicine, Sapporo, Japan and ${ }^{4}$ Department of Geriatric Medicine and Nephrology, Osaka University Medical School, Suita, Japan

Correspondence: Dr K Fukuo, Department of Food Sciences and Nutrition, School of Human Environmental Sciences, Mukogawa Women's University, Nishinomiya, Hyogo, 6638558, Japan.

E-mail: fukuo@mukogawa-u.ac.jp

Received 19 December 2008; revised 25 May 2009; accepted 23 June 2009; published online 21 August 2009 
with control mice. ${ }^{13}$ Another study reported that a high-fat meal did not elicit changes in serum adiponectin compared with fasting baseline levels in healthy male subjects. ${ }^{14}$ Thus, the effects of dietary fat intake on plasma adiponectin concentrations are unclear at present.

It is well established that atherosclerosis is a progressive multifactorial process that begins in early life. ${ }^{15-17}$ However, few studies have examined the risk factors of atherosclerosis development in young women. In particular, the interaction between genes and environmental factors in the development of early atherosclerosis in young female populations is not clear. Thus, this study was designed to investigate the association between $A T 1 R$ A1166C and plasma adiponectin concentrations in young female subjects. We also investigated the influence of AT1R A1166C on the correlation between dietary fat intake and plasma adiponectin concentrations in these subjects.

\section{METHODS}

\section{Participants}

Between July 2004 and December 2007, 416 students from 18 to 23 years of age were recruited from Mukogawa Women's University. We received approval from the Institutional Review Board and written informed consent was obtained from all participants in this study.

\section{Body composition and biochemical measurements}

Blood samples were taken from each of the participants in the morning following overnight fasting. We measured the fasting plasma glucose, insulin, hemoglobin Alc (HbAlc), lipoprotein lipase (LPL), high-sensitivity C-reactive protein (hs-CRP), free fatty acid (FFA) and serum lipid concentrations including triglycerides (TG), high-density lipoprotein (HDL) cholesterol and low-density lipoprotein (LDL) cholesterol by standard laboratory techniques. Plasma levels of adiponectin and plasminogen-activator inhibitor 1 (PAI-1) were measured with commercial kits. Body composition was determined by dual-energy X-ray absorptiometry. Blood pressure was measured between 0900 and $1200 \mathrm{~h}$ (noon), with participants resting in the sitting position. Intimamedia thickness (IMT) in the carotid artery was measured with the participants in a supine position after a few minutes of rest. Indices of obesity and insulin resistance were derived from body mass index (BMI) and a homeostasis model assessment of insulin resistance (HOMA-IR), respectively.

\section{Estimation of habitual food intake}

Percentages of protein, fat and carbohydrate in the diet were estimated by using the responses to a self-administered diet history questionnaire, developed for use in the evaluation of nutrient intake levels and health education among both healthy individuals and high-risk populations. The diet history questionnaire was designed to obtain information about dietary habits for the previous month with regard to total energy and 17 nutrients. ${ }^{18}$

\section{Determination of AT1R A1166C polymorphism genotypes}

Genomic DNA was extracted from $200 \mu \mathrm{l}$ of whole blood, following the blood spin protocol recommended by the manufacturer for use of the QIAamp DNA Blood Kit (Qiagen Inc., Valencia, CA, USA). AT1R A1166C polymorphism genotypes were determined by the TaqMan polymerase chain reaction method. The primers and probes for genotype determinations were as follows: forward primer 5'-CATTCCTCTGCAGCACTTCACT-3', reverse primer 5'-CGGTTC AGTCCACATAATGCAT-3', probe for A (1166) 5'-FAM-CAAATGAGCAT TAGCTACMGB-3', probe for C (1166) 5'-VIC-CAAATGAGCCTTAGCTACTMGB-3'. Each reaction included $1 \mu \mathrm{l}$ of the DNA sample including $30 \mathrm{ng}$ of genetic DNA, $0.3 \mu \mathrm{l}$ of each primer, $0.1 \mu \mathrm{l}$ of each probe, $5 \mu \mathrm{l}$ of the TaqMan Universal Master Mix (Applied Biosystems, Foster City, CA, USA) and $3.2 \mu \mathrm{l}$ of sterile distilled water. Polymerase chain reaction cycling conditions were 1 cycle at $95^{\circ} \mathrm{C}$ for $10 \mathrm{~min}, 45$ cycles at $92^{\circ} \mathrm{C}$ for $15 \mathrm{~s}$ and $60^{\circ} \mathrm{C}$ for $1 \mathrm{~min}$. Polymerase chain reaction and allelic detection were carried out using an ABI Sequence Detection System 7500 and allelic discrimination software, both from Applied Biosystems.

\section{Ultrasound studies}

IMT was measured by ultrasonic diagnosis equipment (Shimazu SDU-2200, Shimazu, Tokyo, Japan) that was programmed with IMT software (Intimascope, Media Cross Co. Ltd., Tokyo, Japan) as described. ${ }^{19}$ This software makes it possible to automatically recognize the edges of the internal and the external membranes of the blood vessels and to automatically measure the distance at a sub-pixel level (estimated to be $0.01 \mathrm{~mm}$ ), using a threedimensional polynomial measurement formula.

Carotid artery ultrasonography was performed using a $10-\mathrm{MHz}$ scanning frequency in B mode. One skilled observer, blinded to the subjective data, scanned the vessel in transverse planes. Subjects were examined in a supine position. Images were obtained in the $20 \mathrm{~mm}$ proximal to the origin of the bulb at the far wall of the right common carotid artery. In all subjects examined in this study, no plaque was observed in this segment. Average IMT was the average value of 250 computer-based points in the region.

\section{Statistical analysis}

Agreement with the Hardy-Weinberg equilibrium was tested by comparing the observed and expected genotype frequencies of the participants using the $\chi^{2}$-test for good fit with Fisher's exact tests. On account of the very low frequency of the $1166 \mathrm{C}$ allele, participants who were homozygous for CC were combined in the analysis with those who were heterozygous AC to increase the statistical power. Normality of variable distribution was tested by the Kolmogorov-Smirnov test.

Quantitative data with a normal distribution were presented as the mean \pm s.d., and data with a skewed distribution were presented as the median (interquartile range). Comparisons between carriers of the $\mathrm{C}$ allele and noncarriers were made using unpaired $t$-tests for continuous variables with normal distribution and with the nonparametric Mann-Whitney $U$-test for continuous variables with a skewed distribution. Pearson's and Spearman's coefficients of correlation were used as appropriate. A univariate generalized linear model was used to test for the heterogeneity of regression. To examine the independent contribution of the AT1R A1166C polymorphism to plasma adiponectin levels when adjusting for the effects of other clinical characteristics, logistic regression analysis with forward stepwise selection was used. For this analysis, participants were divided into two groups at the median of plasma adiponectin concentrations. We adjusted for confounders with the use of analysis of covariance (ANCOVA). To normalize variables with skewed distributions, logarithm, square root and inverse were used for data transformations as appropriate. Data were analyzed with SPSS (release 16.0J; SPSS Japan, Tokyo, Japan) and differences were considered significant with a $P$-value of $\leqslant 0.05$.

\section{RESULTS}

\section{Genotype and participant characteristics}

We examined the influence of $A T 1 R$ A1166C on anthropometric features. The distributions of AT1R A1166C genotypes were as follows: AT1R A1166C AA, $83.2 \%(n=346)$; AC, $15.9 \%$ ( $n=66)$; and CC, $0.9 \%$ $(n=4)$. The observed genotype frequencies in this study were in the Hardy-Weinberg equilibrium $\left(\chi^{2}=0.185, P=0.912\right)$ and were similar to results from previous studies of Japanese populations. ${ }^{20}$ There were no significant differences between carriers of the $\mathrm{C}$ allele and noncarriers in terms of age, height, weight, BMI, SBP, DBP, pulse rate, trunk fat mass, plasma concentrations of lipoproteins, plasma fasting glucose levels, HOMA-IR, HbA1c or IMT (Table 1). However, plasma adiponectin concentrations were significantly lower in carriers of the $\mathrm{C}$ allele compared with non-carriers. For comparison of plasma adiponectin concentrations between genotype groups, we used ANCOVA with BMI, HDL-Ch and HOMA-IR as covariates. The results of the evaluation of the assumptions of normality for sampling distributions and the homogeneity of variance were satisfactory. After adjustment by covariates, plasma adiponectin levels were significantly lower in carriers of the $\mathrm{C}$ allele compared with non-carriers $(P=0.043)$ (Table 2). As shown in Table 3, multiple logistic regression analysis with forward stepwise selection, after controlling for age, BMI, 
Table 1 Characteristics and plasma adipocytokine concentrations of carriers and non-carriers of the C allele of the AT1R A1166C polymorphism

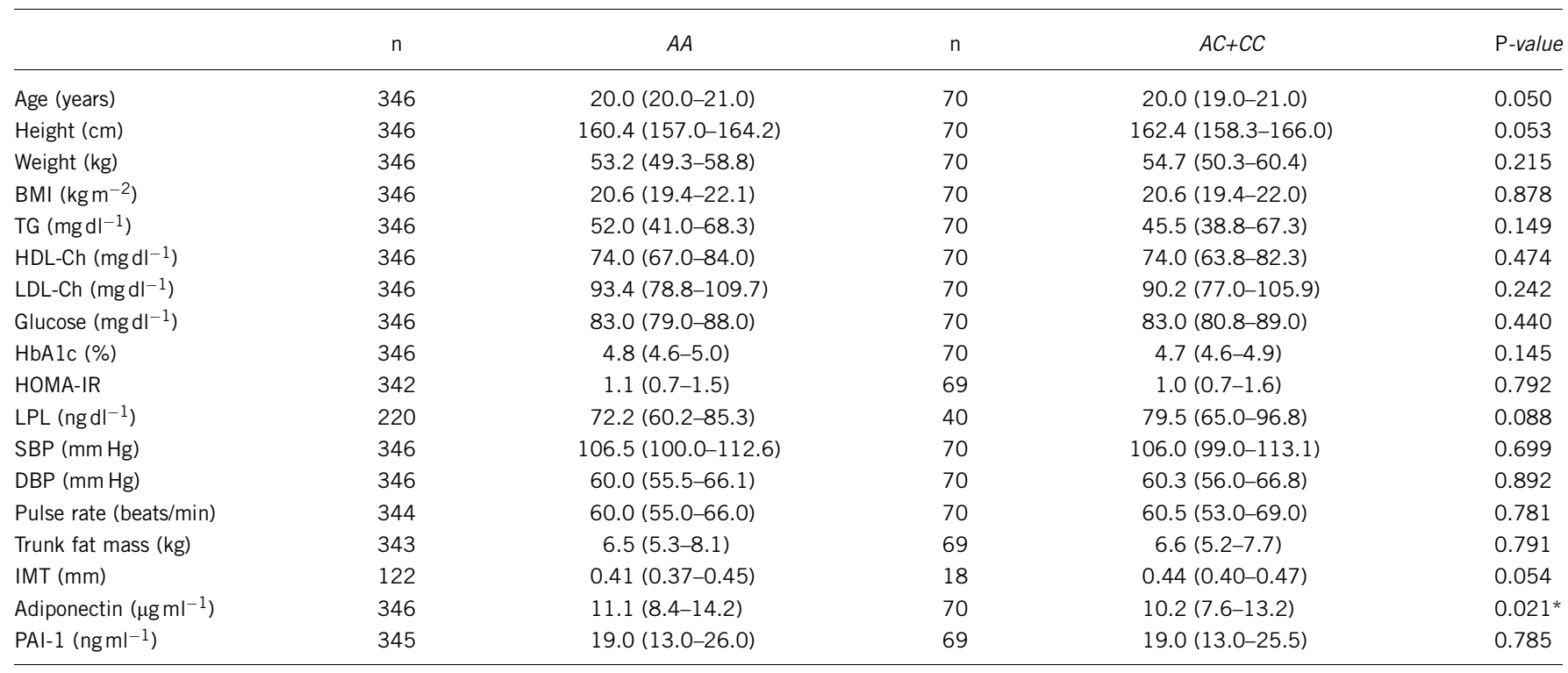

Abbreviations: BMI, body mass index; DBP, diastolic blood pressure; HDL, high-density lipoprotein, HOMA, homeostasis model assessment; IMT, Intima-media thickness; LDL, low-density lipoprotein; PAI-1, plasminogen-activator inhibitor 1; SBP, systolic blood pressure. Values are expressed as median (interquartile range). ${ }^{*} P<0.05$.

Table 2 Analysis of covariance of genotype groups of AT1R A1166C toward plasma adiponectin concentration, with BMI, HDL-Ch and HOMA-IR as the covariates

\begin{tabular}{lccccc}
\hline Source of variance & $\begin{array}{c}\text { Adjusted sum } \\
\text { of square }\end{array}$ & d.f. & F & B & P-value \\
\hline BMI & 3.184 & 1 & 8.567 & 17.187 & $0.004^{* *}$ \\
HDL-Ch & 13.164 & 1 & 35.459 & 0.229 & $<0.001^{* *}$ \\
HOMAIR & 0.088 & 1 & 0.264 & -0.061 & 0.608 \\
Genotype & 1.531 & 1 & 4.123 & -0.164 & $0.043^{*}$ \\
\hline
\end{tabular}

Abbreviations: BMI, body mass index; HDL, high-density lipoprotein; HOMA, homeostasis model assessment.

Data transformation: BMI; Inverse, HDL-Ch; Square root, HOMA-IR; Logarithm. d.f.: degree of freedom, F:F value, $B$ : standardized partial regression coefficient. ${ }^{*} P<0.05,{ }^{* *} P<0.01$. Values are expressed as median (interquartile range).

Table 3 Stepwise logistic regression analysis with above and below the median of plasma adiponectin concentrations as the dependent variable and selected variables as the independent variable

\begin{tabular}{lrrrrrr}
\hline & & & & & \multicolumn{3}{c}{$\begin{array}{c}\text { 95\% Confidence interval } \\
\text { for odds ratio }\end{array}$} \\
\cline { 5 - 7 } Variables & B & s.e. & P-value & Odds ratio & Lower & Upper \\
\hline C allele & 0.720 & 0.291 & 0.010 & 2.055 & 1.184 & 3.565 \\
BMI & 0.104 & 0.045 & 0.022 & 1.110 & 1.015 & 1.213 \\
HDL-Ch & -0.036 & 0.008 & $<0.001$ & 0.965 & 0.950 & 0.980 \\
\hline
\end{tabular}

Abbreviations: BMI, body mass index; HDL, high density lipoprotein; TG, triglycerides. B: partial regression coefficient. Not accepted variables were age, TG, HOMA-IR.

HDL-Ch, triglycerides and HOMA-IR, showed that the genotype with the $\mathrm{C}$ allele of the AT1R A1166C polymorphism was the strongest and the most independent determinant of lower plasma adiponectin concentrations (odds ratio: 2.055, 95\% CI: 1.184-3.565, $P=0.010$ ), followed by BMI (odds ratio: 1.110, 95\% CI: $1.015-1.213, P=0.022$ ) and HDL-Ch (odds ratio: 0.965, 95\% CI: $0.950-0.980, P<0.001$ ). There were no significant differences, however, in plasma PAI-1 concentrations between these two groups.

Next, we examined whether clinical parameters differed between the participants in the lowest quartile of plasma adiponectin concentrations and those in the highest quartile. The participants in the lowest quartile of plasma adiponectin concentration showed significantly lower HDL-Ch and LPL levels and higher BMI and trunk fat mass when compared with participants in the highest quartile. Importantly, carotid IMT levels were significantly higher in the lowest quartile of plasma adiponectin concentrations than those in the highest quartile. No significant differences in low-density lipoprotein cholesterol, triglycerides, HOMA-IR and blood pressure, however, were observed between these groups (Table 4).

Correlations between percentage of fat in the diet and plasma adiponectin concentrations in the genotype groups

Next, we examined the effects of dietary fat intake on plasma adiponectin concentrations in both genotypes. There were no significant differences in the mean or median intake of protein, fat and carbohydrate in the diet between carriers of the $\mathrm{C}$ allele and non-carriers (Table 5). Unexpectedly, a weak but significant positive correlation was found between the estimated percentages of fat in the diet and plasma adiponectin concentrations in non-carriers of the $\mathrm{C}$ allele $(r=0.161, P=0.004)$ but not in carriers $(r=0.148, P=0.263)$. There was no significant difference between the slopes of regression for carriers and non-carriers of the $\mathrm{C}$ allele $(P=0.731)$ (Figure 1$)$.

\section{DISCUSSION}

In this study, we found that plasma adiponectin concentrations were significantly lower in carriers of the C allele of the AT1R A1166C gene polymorphism compared with non-carriers among a population of young Japanese women. To our knowledge, this report is the first to 
Table 4 Characteristics of the participants in the lowest or highest quartiles of plasma adiponectin concentrations

\begin{tabular}{|c|c|c|c|c|c|}
\hline & \multicolumn{4}{|c|}{ Plasma adiponectin concentrations } & \multirow[b]{2}{*}{ P-value } \\
\hline & $n$ & The lowest quartile $<8.3$ & $\mathrm{n}$ & The highest quartile $13.9 \leqslant$ & \\
\hline Trunk fat mass (kg) & 104 & $7.1(5.7-9.4)$ & 104 & $6.5(5.3-7.4)$ & $0.003^{* *}$ \\
\hline HDL-ch $\left(\mathrm{mg} \mathrm{dl}^{-1}\right)$ & 104 & $69.0(61.0-78.0)$ & 105 & $78.0(68.0-90.0)$ & $<0.001^{* *}$ \\
\hline LDL-ch (mgdl-1) & 104 & $93.5 \pm 22.4$ & 105 & $93.0 \pm 23.1$ & 0.869 \\
\hline HOMA-IR & 102 & $1.06(0.74-1.67)$ & 104 & $1.09(0.73-1.55)$ & 0.730 \\
\hline Glucose (mg dl $\left.{ }^{-1}\right)$ & 104 & $83.7 \pm 6.5$ & 105 & $83.3 \pm 7.8$ & 0.747 \\
\hline HbAlc (\%) & 104 & $4.8(4.6-5.0)$ & 105 & $4.7(4.6-4.9)$ & 0.390 \\
\hline Leptin (ng ml-1) & 104 & $8.1(5.3-10.9)$ & 105 & $6.9(5.2-9.5)$ & $0.049 *$ \\
\hline $\mathrm{SBP}(\mathrm{mm} \mathrm{Hg})$ & 104 & $106.0(99.6-114.8)$ & 105 & $108.0(102.5-113.0)$ & 0.409 \\
\hline $\mathrm{DBP}(\mathrm{mm} \mathrm{Hg})$ & 104 & $61.8(55.1-67.4)$ & 105 & $62.5(57.0-68.0)$ & 0.260 \\
\hline
\end{tabular}

Abbreviations: BMI, body mass index; DBP, diastolic blood pressure; HbAlc, hemoglobin Alc; HDL, high-density lipoprotein, HOMA, homeostasis model assessment; IMT, Intima-media thickness; LDL, low-density lipoprotein; LPL, lipoprotein lipase; SBP, systolic blood pressure; TG, triglycerides.

Values are expressed as median (interquartile range) or mean \pm s.d. ${ }^{*} P<0.05,{ }^{*} P<0.01$.

Table 5 Estimated habitual food intakes in carriers and non-carriers of the C allele of the AT1R A1166C polymorphism

\begin{tabular}{|c|c|c|c|c|c|}
\hline & $n$ & $A A$ & $n$ & $A C+C C$ & P-value \\
\hline Fat (percentage of energy) & 309 & $30.1(26.0-33.2)$ & 59 & $30.4(27.0-33.6)$ & 0.852 \\
\hline Carbohydrate (percentage of energy) & 309 & $54.8(51.0-58.9)$ & 59 & $55.5(50.7-58.9)$ & 0.996 \\
\hline Protein (percentage of energy) & 309 & $13.3 \pm 2.0$ & 59 & $13.2 \pm 2.3$ & 0.803 \\
\hline
\end{tabular}

Values are expressed as median (interquartile range) or mean \pm s.d. ${ }^{*} P<0.05$.

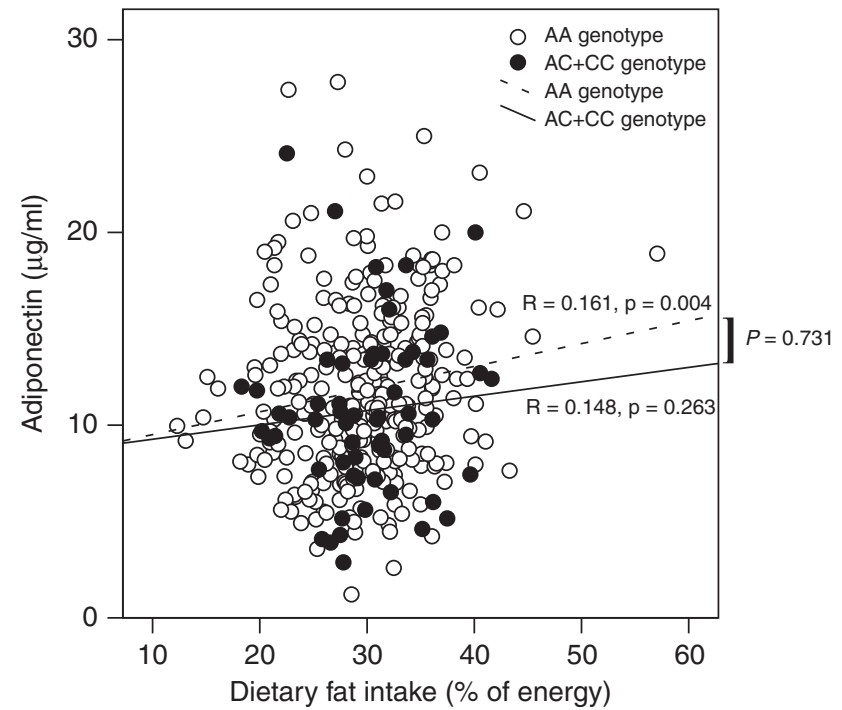

Figure 1 Correlations between dietary fat intake (percentage of energy) and plasma concentrations of adiponectin.

show that the AT1R A1166C polymorphism may influence changes in plasma adiponectin concentrations in humans. Adiponectin is a unique and essential adipocytokine that is produced very abundantly from adipocytes and is stably present in the plasma at very high concentrations. Recent studies emphasize the part played by adiponectin in the homeostasis of adipose tissue and in the pathogenesis of the metabolic syndrome and atherosclerosis. ${ }^{21}$ Adiponectin levels are positively correlated with HDL-cholesterol but inversely with insulin; the levels are reduced in obesity and type II diabetes ${ }^{9}$ and lower in hypertensive patients compared with normotensive patients. ${ }^{22}$ Plasma concentrations of adiponectin are, however, stable throughout the menstrual cycle ${ }^{23}$ and also not affected by menopause in healthy women. ${ }^{24}$

Interestingly, participants in the lowest quartile of plasma adiponectin concentration had lower HDL-Ch and LPL levels and higher trunk fat mass compared with those in the highest quartile. In addition, the participants in the lowest quartile had thicker carotid IMT levels compared with those in the highest quartile. These results suggest that carriers of the $\mathrm{C}$ allele with lower adiponectin concentrations may be more susceptible to the development of atherosclerosis.

Although the mechanism underlying lower adiponectin concentrations in carriers of the $\mathrm{C}$ allele of the AT1R A1166C gene polymorphism is not clear at present, a silent polymorphism, A1166C, occurs in the 3 '-untranslated region of the human AT1R gene that can lead to increased AT1R densities. ${ }^{7}$ This suggests that the effect of AII may be enhanced in carriers of the C allele of the AT1R A1166C polymorphism. ${ }^{4,25-27}$ It is noted that AII infusion decreases plasma adiponectin levels through its type 1 receptor in rats, ${ }^{10}$ and treatment with an AII type 1 receptor antagonist increases plasma adiponectin levels in hypertensive patients. ${ }^{12}$ These results suggest that a difference in activation of the local RAS may be directly involved in the variation between genotypes with regard to plasma adiponectin concentrations. However, further investigation is required to confirm this hypothesis. 
In this study, we also found a genotype-dependent correlation between the percentage of fat in the diet and plasma adiponectin concentrations. Unexpectedly, there was a weak but significant positive correlation between plasma adiponectin concentrations and percentage of fat in the diet in non-carriers of the $\mathrm{C}$ allele, but not in carriers. Although the mechanism of this result is not clear, it was reported earlier that no correlation was found between dietary fat intake and plasma adiponectin concentration in humans, ${ }^{14}$ suggesting that this relation may be specific for this genotype. Follow-up studies are necessary to clarify these points.

In conclusion, we showed that plasma adiponectin concentrations were associated with the $\mathrm{C}$ allele of the $A T 1 R$ gene in young healthy women and that the $A T 1 R$ genotype might affect plasma levels of adiponectin when these young women consume a high-fat diet. These results suggest that genotyping of the AT1R A1166C polymorphism may help to prevent the development of metabolic syndrome and atherosclerosis in young women.

\section{ACKNOWLEDGEMENTS}

We thank Ms Sayaka Ohashi, Ms Kazuko Iwasa, Ms Seiko Kaji and Ms Eriko Nagata for laboratory and technical support during this investigation. This study was supported by a Grant-in-Aid for Scientific Research from the Japanese Ministry of Education, Science, Sports and Culture; also by the Open Research Center Project of Mukogawa Women's University for studying lifestyle-related diseases; by a Grant-in-Aid for Scientific Research (H17-pharmaco-common-003) from the Japanese Ministry of Health, Labor, and Welfare; Grants-in-Aid for Scientific Research (18590265, 18590811, 19650188) from the Ministry of Education, Science, Sports and Culture of Japan; and by research grants from the Takeda Science Foundation and the Japan Research Foundation for Clinical Pharmacology.

1 Prasad A, Quiyyumi AA. Renin-angiotensin system and angiotensin receptor blockers in the metabolic syndrome. Circulation 2004; 110: 1507-1512.

2 Ogihara T, Asano T, Ando K, Chiba Y, Sakoda H, Anai M, Shojima N, Ono H, Onishi Y, Fujishiro M, Katagiri H, Fukushima Y, Kikuchi M, Noguchi N, Aburatani H, Komuro I, Fujita T. Angiotensin II-induced insulin resistance is associated with enhanced insulin signaling. Hypertension 2002; 40: 872-879.

3 Engeli S, Negrel R, Sharma AM. Physiology and pathophysiology of the adipose tissue renin-angiotensin system. Hypertension 2000; 35: 1270-1277.

4 Spiering W, Kroon AA, Fuss-Lejeune MMJJ, Daemen MJAP, de Leeuw PW. Angiotensin II sensitivity is associated with the angiotensin II type 1 receptor A1 166C polymorphism in essential hypertensives on a high sodium diet. Hypertension 2000; 36: 411-416.

5 Bonnardeaux A, Davies E, Jeunemaitre X, Charru A, Clause E, Tiret L, Cambien F, Corvol $\mathrm{P}$, Soubrier F. Angiotensin II type 1 receptor gene polymorphisms in human essential hypertension. Hypertension 1994; 24: 63-69.

6 Abdlooahi MR, Gaunt TR, Syddall HE, Cooper C, Phillips DIW, Ye S, Day INM. Angiotensin II type I receptor gene polymorphism: anthropometric and metabolic syndrome traits. J Med Genet 2005; 42: 396-401.

7 Martin MM, Buckenberger JA, Jiang J, Malana GE, Nuovo GJ, Chotani M, Feldman DS, Schmittgen TD, Elton TS. The human angiotensin II type 1 receptor +1166 A/C polymorphism attenuates microRNA-155 binding. J Biol Chem 2007; 282 24262-24269.
8 Jaleel F, Jaleel A, Aftab J, Rahman MA. Relationship between adiponectin, glycemic control and blood lipids in diabetic type 2 postmenopausal women with and without complication of ischemic heart disease. Clin Chim Acta 2006; 370: 76-81.

9 Mahmud A, Feely J. Adiponectin and arterial stiffness. Am J Hypertens 2005; 18 : 1543-1548.

10 Ran J, Hirano T, Fukui T, Saito K, Kageyama H, Okada K, Adachi M. Angiotensin II infusion decreases plasma adiponectin level via its type 1 receptor in rats: an implication for hypertension-related insulin resistance. Metabolism 2006; 55: 478-488.

11 Furuhashi M, Ura N, Takizawa H, Yoshida D, Moniwa N, Murakami H, Higashiura K, Shimamoto K. Blockade of the renin-angiotensin system decreases adipocyte size with improvement in insulin sensitivity. J Hypertens 2004; 22: 1977-1982.

12 Furuhashi M, Ura N, Higashiura K, Murakami H, Tanaka M, Moniwa N, Yoshida D, Shimamoto K. Blockade of the renin-angiotensin system increases adiponectin concentrations in patients with essential hypertension. Hypertension 2003; 42: 76-81.

13 Zhou Q, Du J, Hu Z, Walsh K, Wang XH. Evidence for adipose -muscle cross talk: opposing regulation of muscle proteolysis by adiponectin and fatty acids. Endocrinology 2007: 148: 5696-5705.

14 Poppitt SD, Keogh GF, Lithander FE, Wang Y, Mulvey TB, Chan YK, McArdle BH, Cooper GJS. Postprandial response of adiponectin, interleukin-6, tumor necrosis factor$\alpha$, and C-reactive protein to a high-fat dietary load. Nutrition 2008; 24: 322-329.

15 Zieske AW, Tracy RP, McMahan CA, Herderick EE, Homma S, Malcom GT, Mcgill HC, Strong JP. Elevated serum C-reactive protein levels and advanced atherosclerosis in youth. Arterioscler Thromb Vac Biol 2005; 25: 1237-1243.

16 Raitakari OT, Juonala M, Kahonen M, Taottonen L, Laitinen T, Maki-Torkko N, Jarvisalo MJ, Uhari M, Jokinen E, Ronnemaa T, Akerblom HK, Viikari JS. Cardiovascular risk factors in childhood and carotid artery intima-media thickness in adulthood. JAMA 2003; 290: 2277-2283.

17 Knoflach M, Kiechl S, Kind M, Said M, Sief R, Gisinger M, van der Zee R, Gaston H, Jarosch E, Willeit J, Wick G. Cardiovascular risk factors and atherosclerosis in young males: ARMY study. Circulation 2003; 108: 1064-1069.

18 Sasaki S, Yanagibori R, Amano K. Validity of a self-administered diet history questionnaire for assessment of sodium and potassium -Comparison with single 24-h urinary excretion. Jpn Circ J 1998; 62: 431-435.

19 Yanase T, Nasu S, Mukuta Y, Shimizu Y, Nishihara T, Okabe T, Nomura M, Inoguchi T, Nawata $\mathrm{H}$. Evaluation of a new carotid intima-media thickness measurement by B-mode ultrasonography using an innovative measurement software, intimascope. Am J Hypertens 2006; 19: 1206-1212.

20 Kikuya M, Sugimoto K, Katsuya T, Suzuki M, Sato T, Funahashi J, Katoh R, Kazama I, Michimata M, Araki T, Hozawa A, Tsuji I, Ogihara T, Yanagisawa T, Imai Y, Mtsubara M. A/C1166 gene polymorphism of the angiotensin II type 1 receptor (AT1) and ambulatory blood pressure: the Ohasama Study. Hypertens Res 2003; 26: 141-145.

21 Matsuzawa Y, Funahashi T, Kihara S, Shimomura I. Adiponectin and metabolic syndrome. Arterioscler Thromb Vasc Biol 2004; 24: 29-33.

22 Tsuda K. Adiponectin and membrane fluidity of erythrocytes in normotensive and hypertensive men. Obesity 2006; 14: 1505-1510.

23 Asimakopoulos B, Milousis A, Gioka T, Kabouromiti G, Gianisslis G, Troussa A, Simopoulou M, Katergari S, Tripsianis G, Nikolettos N. Serum pattern of circulation adipokines throughout the physiological menstrual cycle. Endocr. J 2009; Feb: 18.

24 Muscelli E, Kozakova M, Flyvbjerg A, Kyriakopoulou K, Astiarraga BD, Glintborg D, Konrad T, Favuzzi A, Petrie J. The effect of menopause on carotid artery remodeling, insulin sensitivity, and plasma adiponectin in healthy women. Am J Hypertens 2009; 22: 354-370.

25 Van Geel PP, Pinto YM, Voors AA, Buikema H, Oosterga M, Crijns HJGM, van Gilst WH. Angiotensin II type 1 receptor $\mathrm{A} 1166 \mathrm{C}$ gene polymorphism is associated with an increased response to angiotensin II in human arteries. Hypertension 2000; 35: 717-721.

26 Nishikino M, Matsunaga T, Yasuda K, Adachi T, Moritani T, Tsujimoto G, Tsuda K, Aoki N. Genetic variation in the renin-angiotensin system and autonomic nervous system function in young healthy Japanese subjects. J Clin Endocrinol Metab 2006; 91: 4676-4681.

27 Lim HS, Cho JY, Oh DS, Chung JY, Hong KS, Bae KS, Yu KS, Lee KH, Jang IJ, Shin SG. Angiotensin II type 1 receptor $1166 \mathrm{~A} / \mathrm{C}$ polymorphism in association with blood pressure response to exogenous angiotensin II. Eur J Clin Pharmacol 2007; 63: 17-26. 